\title{
Experimental effects of acute exercise on forgetting
}

\author{
D.C. MOORE, S. RYU and P.D. LOPRINZI* (1)
}

Department of Health, Exercise Science and Recreation Management, Exercise \& Memory Laboratory, The University of Mississippi, University, MS 38677, USA

Received: January 11, 2020 • Accepted: July 7, 2020

Published online: September 29, 2020

(c) 2020 Akadémiai Kiadó, Budapest

\begin{abstract}
Objective: Prior research has evaluated the effects of acute exercise on episodic memory function. These studies have, on occasion, demonstrated that acute exercise may enhance both short- and long-term memory. It is uncertain as to whether the acute exercise improvements in long-term memory are a result of acute exercise attenuating declines in long-term memory, or rather, are driven by the enhancement effects of acute exercise on short-term memory. The present empirical study evaluates whether the decline from short- to long-term is influenced by acute exercise. This relationship is plausible as exercise has been shown to activate neurophysiological pathways (e.g., RAC1) that are involved in the mechanisms of forgetting. Methods: To evaluate the effects of acute exercise on forgetting, we used data from 12 of our laboratory's prior experiments $(N=538)$. Across these 12 experiments, acute exercise ranged from 10 to 15 mins in duration (moderate-to-vigorous intensity). Episodic memory was assessed from word-list or paragraphbased assessments. Short-term memory was assessed immediately after encoding, with long-term memory assessed approximately 20 -min later. Forgetting was calculated as the difference in short- and long-term memory performance. Results: Acute exercise (vs. seated control) was not associated with an attenuated forgetting effect $(d=0.10$; $95 \% \mathrm{CI}:-0.04,0.25, P=0.17)$. We observed no evidence of a significant moderation effect $\left(Q=6.16, \mathrm{df}=17, P=0.17, I^{2}=0.00\right)$ for any of the evaluated parameters, including study design, exercise intensity and delay period. Conclusion: Across our 12 experimental studies, acute exercise was not associated with an attenuated forgetting effect. We discuss these implications for future research that evaluates the effects of acute exercise on long-term memory function.
\end{abstract}

* Corresponding author. Department of Health, Exercise Science, and Recreation Management, Exercise \& Memory Laboratory, The University of Mississippi, 229 Turner Center, University, MS 38677, USA. Tel.: +1 662915 5561; fax: +1 662915 5521. E-mail: pdloprin@olemiss.edu 


\section{KEYWORDS}

cognition, memory encoding, memory consolidation, physical activity

\section{INTRODUCTION}

Emerging work demonstrates that acute exercise may enhance short- and long-term episodic memory function [1]. Less investigated, however, is whether acute exercise can attenuate declines in long-term memory. It is uncertain as to whether the acute exercise improvements in long-term memory are a result of acute exercise attenuating declines in long-term memory, or rather, are driven by the enhancement effects of acute exercise on short-term memory. The present empirical study evaluates whether the decline from short- to long-term is influenced by acute exercise, i.e., whether acute exercise can attenuate forgetting. The narrative that follows briefly discusses the neurophysiological mechanisms of forgetting and how they relate and are different than the mechanisms of memory formation. Where appropriate, we also discuss the role of exercise on these mechanisms to indicate the theoretical plausibility through which acute exercise may, potentially, attenuate forgetting.

Forgetting is defined as failure to either recall or retain information into present consciousness [2]. Assuming all experiences leave traces in the brain, failure to retain or reactivate these traces induces forgetting.

The acquisition of information alters the physiological state of neurons responsible for creating the memory trace; ${ }^{1}$ consolidation is the processes of stabilizing a memory trace after its initial acquisition (encoding); and memory retrieval is the process of reactivating the memory trace to induce remembering of the previously encoded information.

The Ebbinghaus Forgetting Curve ${ }^{2}$ [3] displays the balance between the strengthening forces of acquisition and consolidation and the opposing (weakening) forces of forgetting. Memories that are deemed important and worthwhile may remain while allowing the irrelevant ones to be removed by passive or active processes of forgetting. ${ }^{3}$ These processes are thought to be

\footnotetext{
${ }^{1}$ Molecular or cellular memory traces can be, in principle, any change in the activity of the cell that is induced by learning that becomes part of the neural code for that memory.

${ }^{2}$ Mechanisms at fault for the steep decay in retained information over the 20 min after encoding are responsible for the decay of memory traces. The decay process is thought to provide balance to the processes of acquisition and consolidation.

${ }^{3}$ Passive forgetting is described as the biological decay of memory traces due to molecular turnover (natural decay), loss of context cues across time that make retrieval difficult, the accumulation of similar memory traces across time that interfere with proper retrieval, and changes in the memory's context between acquisition and retrieval that impair recall. Active forgetting can be defined as the partial erosion of only some of the molecular and cellular memory trace, or when a portion of the engram cells become disconnected from the engram circuit, thus making the memory engram unresponsive to recall mechanisms. For example, interference-based forgetting suggests that new information presented prior to the learning event (proactive) or after (retroactive) attenuates memory expression by accelerating the memory decay. This may occur due to brain activity and memory formation utilizing the same resources that are used for memory consolidation, thus, limited resources are in competition with one another to form or recall a memory trace. Motivated forgetting may include an emotion-based forgetting process under our own cognitive control. Memories that evoke sadness, guilt, or embarrassment (some unpleasant quality) can strongly influence recalling a memory. If such memories are recalled during encoding or consolidation, suppressing the memories voluntarily may disrupt the process of forming a stable engram, leading to forgetting. Retrieval-induced forgetting occurs when recalling memories causes other related information to be forgotten.
} 
influenced by intrinsic biological pathways responsible for the decay of a memory trace or the engram cell circuit.

Recent research has discussed the neurophysiological mechanisms of acquisition, consolidation, and memory retrieval [4-6]. Less research, however, has elucidated the underlying neurophysiological mechanisms related to forgetting. We discuss this in terms of indirect and direct effects.

Indirect evidence indicates that shared neurophysiological pathways are involved in both memory formation and forgetting. For forgetting, these include, for example, inhibition of NMDA receptors or CAMKII activity, BDNF, cytoplasmic polyadenylation element binding protein (CPEB), and PKM $\zeta$ and GluA2-dependent AMPA receptor trafficking [7]. Notably, exercise, particularly chronic exercise, has been shown to influence the expression of these parameters [8-10], providing plausibility through which exercise may influence forgetting.

Regarding direct effects, the RAC1 pathway is a member of small G proteins that are responsible for influencing the size and shape of synaptic spines by regulating actin polymerization [11]. As such, RAC1 has been implicated in synaptic plasticity and memory [12]. Evidence suggests that RAC1 mediates several types of active forgetting of olfactory memories in Drosophila $^{4}[13,14]$. For example, Jiang et al. (2016) demonstrated that inhibiting RAC1 in the hippocampus increased memory after contextual fear conditioning, while activating RAC1 weakened memory produced by spaced, contextual fear conditioning [15]. Further, Liu et al. (2016) showed that increased activation of RAC1 in the mouse hippocampus accelerated decay of object-recognition by 3-fold [16]. In the context of exercise, recent research demonstrates that exercise regulates pathways involved in forgetting (e.g., RAC1) to attenuate aging-related synaptic loss and forgetting [17]. For example, chronic exercise among rats has been shown to increase synaptophysin levels but lower cofilin levels [17].

Our lab has extensively evaluated the effects of acute exercise on memory function and have, approximately half of the time, demonstrated favorable effects [18]. We have also discussed the potential mechanisms of this relationship, likely influenced from alterations in neuronal excitability and its ensuing long-term potentiation [18-22]. However, very limited research has evaluated the effects of acute exercise on forgetting, which was the purpose of the present paper. As we have recently hypothesized [23], this potential relationship is plausible as acute exercise prior to encoding may promote memory consolidation by enhancing the strength and importance of the memory engram, delaying the intrinsic forgetting processes by attenuating RAC1, or inhibiting intrinsic factors that influence memory (interference-based forgetting, motivated based forgetting, and retrieval-induced forgetting). For this paper, we hypothesize that acute exercise, as compared to a seated control scenario, will be associated with less forgetting. Such an effect, for example, may occur from cognitive (e.g., executive control) and neurophysiological (e.g., RAC1) perspectives. If, however, this effect is not observed, then this will have important implications for research evaluating the effects of acute exercise on long-term memory. That is, if acute exercise is associated with improvements in both short- and long-term memory, but there are no differences in "forgetting" (slope between short- and long-term memory), then

\footnotetext{
${ }^{4}$ Types of forgetting include intrinsic forgetting, interference-induced forgetting, trace memory forgetting, and reversal learning-activated memory (Shuai et al. 2010).
} 
observed acute exercise-induced effects on long-term memory may be driven by exerciseinduced improvements in short-term memory.

\section{MATERIALS \& METHODS}

The present paper is a secondary analysis of 12 of our prior experimental studies that focused on the effects of acute exercise on memory function. For this paper, the outcome of interest was "forgetting". This outcome was calculated as the difference in short- and long-term memory function (i.e., short-term memory minus long-term memory performance). Details of these 12 studies can be found in Table 1. All 12 studies implemented the acute bout of exercise before memory encoding. For each of these 12 studies, either a between-subject randomized controlled design was implemented (exercise vs. control), or a within-subject, counterbalanced design was employed.

\section{Categorization of moderators}

Given the evaluation of 12 experimental studies for this paper, we implement a meta-analytic approach to this paper, which is in direct alignment with recommendations made by Cummings, who indicates that, when feasible, scholars should conduct multiple studies and metaanalyze their laboratory's results [24].

The evaluated moderators included study design, delay period, and exercise intensity. Study design was categorized as a between-subject or within-subject design. For the episodic memory type, we evaluated both short-term and long-term episodic memory, with the latter defined as a delayed period of 20-min or longer. Exercise intensity was based on thresholds suggested by the American College of Sports Medicine [25]. For example, based on maximum heart rate estimates, moderate and vigorous-intensity exercise, respectively, were defined as $64-76 \%$ and $>76 \%$.

\section{Data synthesis}

The Comprehensive Meta-Analysis software (Version 3, Biostat, NJ, USA) was used to calculate effect sizes (Cohen's d) and 95\% CI (see Fig. 1), employing a random-effects model. Effect sizes included the forgetting metric (i.e., short-term memory minus long-term memory performance) between the exercise and control conditions. The weighted mean effect size (Cohen's d) and 95\% CI were calculated using the inverse variance weighting method. Effect size estimates were evaluated for each of the above-mentioned moderators. The degree of heterogeneity of the effect sizes was evaluated with the Cochran's Q-statistic. Egger's regression test was used to evaluate the potential publication bias. In addition to evaluating effect size estimates for forgetting between the two conditions (exercise, control), we calculated short-term memory and long-term memory as a proportion (Figs. 2 and 3). For example, if they recalled $60 \%$ of words for short-term memory and $50 \%$ of words for the long-term memory assessment, the forgetting score was $10 \%$.

\section{RESULTS}

\section{Article synthesis}

Details on the study characteristics are displayed in Table 1 (extraction table). All studies included young adults (18-21 years). Sample sizes ranged from 20 to 88 participants, with the 
Table 1. Characteristics of the 12 evaluated studies

\begin{tabular}{|c|c|c|c|c|c|c|}
\hline Reference & Sample & Study design & $\begin{array}{l}\text { Exercise } \\
\text { temporality }\end{array}$ & Exercise protocol & $\begin{array}{l}\text { Memory } \\
\text { Assessment }\end{array}$ & Results \\
\hline [26] & $\begin{array}{c}40 \text { adult, } \mathrm{M}_{\mathrm{age}} \\
20.8\end{array}$ & Between-subject & $\begin{array}{l}\text { Acute exercise } \\
\text { occurred before } \\
\text { memory test and } \\
\text { delayed recall }\end{array}$ & $\begin{array}{l}15 \text { min of treadmill } \\
\text { walking at } 70 \% \text { of } \\
\text { estimated heart rate }\end{array}$ & $\begin{array}{c}\text { Face-name memory } \\
\text { task }\end{array}$ & $\begin{array}{l}\text { There was no } \\
\text { difference on } \\
\text { memory scores } \\
\text { across the } \\
\text { experimental } \\
\text { groups }\end{array}$ \\
\hline [27] & $\begin{array}{c}24 \text { adults, } \mathrm{M}_{\text {age }} \\
20.9 \pm 1.9\end{array}$ & Within-Subject & $\begin{array}{l}\text { exercise occurred } \\
\text { before memory test } \\
\text { and delayed recall }\end{array}$ & $\begin{array}{l}15 \text { min of treadmill } \\
\text { walking at } 51 \% \text { of } \\
\text { estimated heart rate } \\
\text { followed by } 5 \text { min of } \\
\text { rest }\end{array}$ & RAVLT & $\begin{array}{l}\text { Short-term memory } \\
\text { was greater in the } \\
\text { visit that involved } \\
\text { exercising prior to } \\
\text { memory encoding } \\
(F=3.76 ; P=0.01 \text {; } \\
\left.\eta^{2}=0.79\right) \text {. Similar } \\
\text { results occurred for } \\
\text { long-term memory, } \\
\text { with no significant } \\
\text { findings. }\end{array}$ \\
\hline [28] & $\begin{array}{c}40 \text { adults, } \mathrm{M}_{\text {age }} \\
21.0\end{array}$ & Within-Subject & $\begin{array}{l}\text { exercise occurred } \\
\text { before memory test } \\
\text { and delayed recall }\end{array}$ & $\begin{array}{l}15 \text { min of treadmill } \\
\text { walking at } 70 \% \text { of } \\
\text { estimated heart rate } \\
\text { followed by } 5 \text { min of } \\
\text { rest }\end{array}$ & RAVLT & $\begin{array}{l}\begin{array}{r}\text { Significant main } \\
\text { effect for time }(P< \\
\left.0.001, \eta^{2}{ }_{\mathrm{P}}=0.77\right) \\
\text { (continued) }\end{array}\end{array}$ \\
\hline
\end{tabular}


Table 1. Continued

\begin{tabular}{|c|c|c|c|c|c|c|}
\hline Reference & Sample & Study design & $\begin{array}{c}\text { Exercise } \\
\text { temporality }\end{array}$ & Exercise protocol & $\begin{array}{c}\text { Memory } \\
\text { Assessment }\end{array}$ & Results \\
\hline [29] & $\begin{array}{c}20 \text { adults, } M_{\text {age }} \\
21.6 \pm 0.7\end{array}$ & $\begin{array}{c}\text { Three-arm, } \\
\text { counterbalanced, } \\
\text { within-subject } \\
\text { design }\end{array}$ & $\begin{array}{l}\text { exercise occurred } \\
\text { before memory test } \\
\text { and delayed recall }\end{array}$ & $\begin{array}{c}10 \text { min of exercise } \\
\text { on treadmill at } 70 \% \\
\text { (moderate intensity) } \\
\text { of estimated heart } \\
\text { rate }\end{array}$ & RAVLT & $\begin{array}{l}\text { The exercise } \\
\text { conditions had a } \\
\text { greater learning } \\
\text { effect when } \\
\text { compared to the } \\
\text { control visit, } \mathrm{M}_{\mathrm{diff}} \\
=0.68, P=0.02 \text {. } \\
\text { The exercise }+ \\
\text { memory visit had } \\
\text { better long-term } \\
\text { memory when } \\
\text { compared to } \\
\text { exercise only, } \mathrm{M}_{\mathrm{diff}} \\
=0.095, P=0.03 \text {. }\end{array}$ \\
\hline [30] & $\begin{array}{c}40 \text { adults, } M_{\text {age }} \\
21.1 \pm 1.1\end{array}$ & $\begin{array}{l}\text { Parallel-group, } \\
\text { randomized } \\
\text { controlled between- } \\
\text { subject design }\end{array}$ & $\begin{array}{l}\text { exercise occurred } \\
\text { before memory test } \\
\text { and delayed recall }\end{array}$ & $\begin{array}{c}15 \text { min of exercise } \\
\text { on treadmill at } 70 \% \\
\text { (moderate intensity) } \\
\text { of estimated heart } \\
\text { rate }\end{array}$ & $\begin{array}{l}\text { Prioritized learning } \\
\text { task. Paragraph } \\
\text { recall, with } \\
\text { participants reading } \\
\text { four sentences and } \\
\text { recalling as much } \\
\text { information as } \\
\text { possible from the } \\
\text { paragraph }\end{array}$ & $\begin{array}{l}\text { Acute exercise did } \\
\text { not improve } \\
\text { neutral-based and } \\
\text { emotional memory } \\
\text { function. }\end{array}$ \\
\hline
\end{tabular}




\section{Table 1. Continued}

\begin{tabular}{|c|c|c|c|c|c|c|}
\hline Reference & Sample & Study design & $\begin{array}{l}\text { Exercise } \\
\text { temporality }\end{array}$ & Exercise protocol & $\begin{array}{c}\text { Memory } \\
\text { Assessment }\end{array}$ & Results \\
\hline [31] & $\begin{array}{l}34 \text { adult females } \\
\mathrm{M}_{\text {age }} 20.65 \pm 1.5\end{array}$ & $\begin{array}{l}\text { Two-arm, parallel- } \\
\text { group (between- } \\
\text { subject) randomized } \\
\text { controlled } \\
\text { experiment }\end{array}$ & $\begin{array}{l}\text { exercise occurred } \\
\text { before memory test } \\
\text { and delayed recall }\end{array}$ & $\begin{array}{l}15 \text { min of treadmill } \\
\text { walking at } 64 \% \\
\text { (moderate intensity) } \\
\text { of estimated heart } \\
\text { rate followed by } 15 \\
\text { min of rest }\end{array}$ & 50 item IAPS images & $\begin{array}{c}\text { There was } \\
\text { significant main } \\
\text { effect for time }(F(2) \\
=104.2, P<0.001 \text {, } \\
\left.\eta_{\mathrm{P}}^{2}=0.77\right) \text { and a } \\
\text { significant main } \\
\text { effect for valence- } \\
\text { arousal } \\
\text { classification }(F(4) \\
=21.39, P<0.001, \\
\left.\eta_{\mathrm{P}}^{2}=0.40\right) .\end{array}$ \\
\hline [32] & $\begin{array}{c}40 \text { adults, } \mathrm{M}_{\text {age }} \\
20.9 \pm 0.95\end{array}$ & $\begin{array}{l}\text { Two-arm, parallel- } \\
\text { group (between- } \\
\text { subject) randomized } \\
\text { controlled } \\
\text { experiment }\end{array}$ & $\begin{array}{l}\text { exercise occurred } \\
\text { before memory test } \\
\text { and delayed recall }\end{array}$ & $\begin{array}{l}15 \text { min of treadmill } \\
\text { walking at } 64 \% \\
\text { (moderate intensity) } \\
\text { of estimated heart } \\
\text { rate followed by } 15 \\
\text { min of rest }\end{array}$ & $\begin{array}{l}\text { Prioritized learning } \\
\text { task. Paragraph } \\
\text { recall, with } \\
\text { participants reading } \\
\text { six paragraphs and } \\
\text { recalling as much } \\
\text { information as } \\
\text { possible from the } \\
\text { paragraphs }\end{array}$ & $\begin{array}{c}\text { Iconic memory } \\
\text { expressed a } \\
\text { significant main } \\
\text { effect for time }(F= \\
42.9, P=<0.001 \\
\left.\eta_{\mathrm{P}}^{2}=0.53\right) \text { and a } \\
\text { trend towards a } \\
\text { group x time } \\
\text { interaction }(F= \\
2.90, P=0.09, \eta_{\mathrm{P}}^{2} \\
=0.07) \text {, but no } \\
\text { main effect for } \\
\text { group }(F=0.82, P \\
\left.=0.37, \eta_{\mathrm{P}}^{2}=0.02\right) . \\
\text { (continued) }\end{array}$ \\
\hline
\end{tabular}


Table 1. Continued

\begin{tabular}{|c|c|c|c|c|c|c|}
\hline Reference & Sample & Study design & $\begin{array}{l}\text { Exercise } \\
\text { temporality }\end{array}$ & Exercise protocol & $\begin{array}{c}\text { Memory } \\
\text { Assessment }\end{array}$ & Results \\
\hline [33] & $\begin{array}{c}20 \text { adults, } \mathrm{M}_{\text {age }} \\
21.1 \pm 1.0\end{array}$ & $\begin{array}{l}\text { Counterbalanced, } \\
\text { randomized, } \\
\text { controlled, within- } \\
\text { Subject design }\end{array}$ & $\begin{array}{l}\text { exercise occurred } \\
\text { before memory test } \\
\text { and delayed recall }\end{array}$ & $\begin{array}{l}20 \text { min of treadmill } \\
\text { walking at } 59.0 \% \text { of } \\
\text { estimated heart rate } \\
\text { followed by } 5 \text {-min of } \\
\text { recovery }\end{array}$ & $\begin{array}{l}\text { Deese-Roediger- } \\
\text { McDermott (DRM) } \\
\text { Paradigm. Included } \\
\text { a } 15 \text {-item word list }\end{array}$ & $\begin{array}{l}\text { Short-term and } \\
\text { long-term memory } \\
\text { groups that } \\
\text { involved exercise } \\
\text { prior to encoding } \\
\text { resulted in better } \\
\text { short-term and } \\
\text { long-term memory } \\
\text { function }(F(2)= \\
11.56, P<0.001, \eta^{2} \mathrm{P} \\
=0.38) \text {. For both } \\
\text { time points the } \\
\text { control visit resulted } \\
\text { in a greater number } \\
\text { of false memories. } \\
\text { These findings } \\
\text { suggest that acute } \\
\text { moderate-intensity } \\
\text { exercise may help to } \\
\text { increase the } \\
\text { accurate recall of } \\
\text { past episodic } \\
\text { memories and } \\
\text { reduce the rate of } \\
\text { false memories. } \\
\text { (continued) }\end{array}$ \\
\hline
\end{tabular}


Table 1. Continued

\begin{tabular}{|c|c|c|c|c|c|c|}
\hline Reference & Sample & Study design & $\begin{array}{l}\text { Exercise } \\
\text { temporality }\end{array}$ & Exercise protocol & $\begin{array}{c}\text { Memory } \\
\text { Assessment }\end{array}$ & Results \\
\hline [34] & $\begin{array}{c}80 \text { adults, Mage } \\
21.0 \pm 1.2\end{array}$ & $\begin{array}{l}\text { Between-subject } \\
\text { randomized } \\
\text { controlled design }\end{array}$ & $\begin{array}{l}\text { exercise occurred } \\
\text { before memory test } \\
\text { and delayed recall }\end{array}$ & $\begin{array}{l}\text { Across three } \\
\text { experiments, the } \\
\text { experimental group } \\
\text { (exercise only) } \\
\text { exercised for } 15 \text { min } \\
\text { on a treadmill } \\
\text { walking at } 60,77 \text {, } \\
\text { and } 62 \% \text {, } \\
\text { respectively, of } \\
\text { estimated heart rate } \\
\text { followed by } 5 \text {-min of } \\
\text { recovery }\end{array}$ & $\begin{array}{l}\text { Logical Memory } \\
\text { Test - two short } \\
\text { narrative passages } \\
\text { (25 lines long). }\end{array}$ & $\begin{array}{l}\text { Both learning } \\
\text { techniques (3-R } \\
\text { techniques \& cue- } \\
\text { recognition) were } \\
\text { effective in } \\
\text { enhancing memory } \\
\text { and provided } \\
\text { evidence of a main } \\
\text { effect for acute } \\
\text { exercise. }\end{array}$ \\
\hline [35] & $\begin{array}{c}24 \text { adults, Mage } \\
21.04 \pm 1.1\end{array}$ & $\begin{array}{l}\text { Within-Subject, } \\
\text { counterbalanced } \\
\text { randomized } \\
\text { controlled design }\end{array}$ & $\begin{array}{l}\text { exercise occurred } \\
\text { before memory test } \\
\text { and delayed recall }\end{array}$ & $\begin{array}{l}15 \text { min of exercise } \\
\text { on treadmill at } 50 \% \\
\text { (moderate intensity) } \\
\text { of heart rate reserve }\end{array}$ & $\begin{array}{l}\text { Word-list memory } \\
\text { task, computer- } \\
\text { based episodic } \\
\text { memory task } \\
\text { (Treasure Hunt } \\
\text { Task), and a } \\
\text { computer-based } \\
\text { planning task } \\
\text { (Tower of London). }\end{array}$ & $\begin{array}{l}\text { No differences were } \\
\text { observed in } \\
\text { objective cognitive } \\
\text { performance or } \\
\text { outcome } \\
\text { expectations. }\end{array}$ \\
\hline
\end{tabular}




\begin{tabular}{cccc}
\hline Reference & Sample & Study design & $\begin{array}{c}\text { Exercise } \\
\text { temporality }\end{array}$ \\
\hline$[36]$ & $\begin{array}{c}\text { 88 young adults, } \\
\text { Mage 21.9 } \pm 2.4\end{array}$ & $\begin{array}{c}\text { Within-Subject } \\
\text { randomized } \\
\text { controlled design }\end{array}$ & $\begin{array}{c}\text { exercise occurred } \\
\text { before memory test } \\
\text { and delayed recall }\end{array}$ \\
& & & \\
{$[37]$} & & & \\
& & & \\
& Mage 23.3 \pm 3.7 & Within-subject & quasi-experimental \\
design & $\begin{array}{c}\text { exercise occurred } \\
\text { before memory test } \\
\text { and delayed recall }\end{array}$
\end{tabular}

$\begin{array}{lr}\text { Exercise protocol } & \begin{array}{c}\text { Memory } \\ \text { Assessmen }\end{array} \\ 15 \text { min of jogging on } & \text { RAVLT } \\ \text { a treadmill with the } \\ \text { first } 5 \text { min at a light } \\ \text { intensity, the next } 5 \\ \text { min at a moderate } \\ \text { intensity, and the } \\ \text { last } 5 \text { min at a } \\ \text { vigorous intensity } \\ \text { (92\% of heart rate } \\ \text { max). } \\ 15 \text { min of exercise } \\ \text { on the treadmill at } \\ 58 \% \text { of maximal } \\ \text { heart rate with an } \\ \text { average speed of } 3.2 \\ \text { mph }\end{array}$

Results
High-intensity
exercise prior to
memory encoding
was effective in
enhancing long-
term memory.




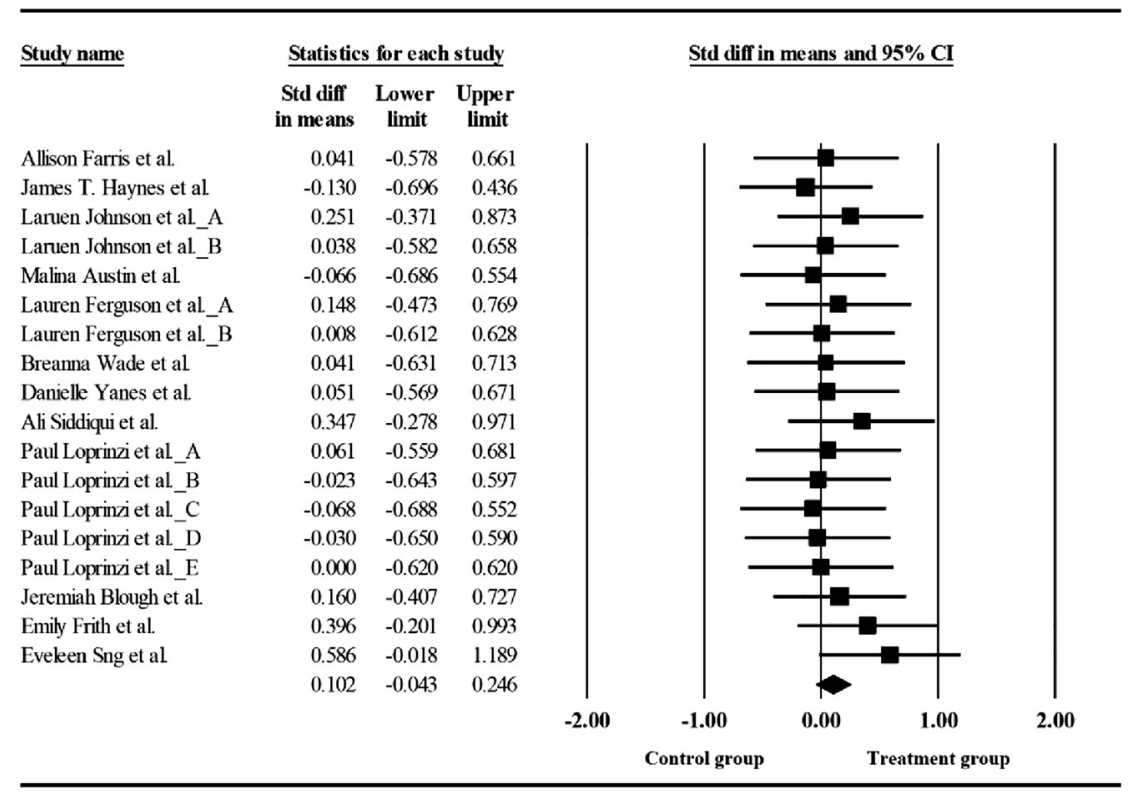

Fig. 1. Forest plot showing standardized mean difference effect sizes and their associated $95 \%$ confidence interval.

total sample size of this analysis being 538. Among the 12 studies, 4 (33\%) employed a betweensubject design and $8(67 \%)$ employed a within subject design. The acute exercise protocols varied, including 10-min of moderate exercise, 15-min brisk walk, and 15-min of high-intensity exercise. Common memory assessments included word list formats (e.g., RAVLT; Rey Auditory Verbal Learning Test) and paragraph recalls, but other episodic memory assessments included, for example, image recognition, DRM (Deese-Roediger-McDermott) and a face name memory task.

\section{Quantitative results}

Fig. 1 displays the overall meta-analytic results (effect size) regarding the effects of acute exercise on forgetting. This overall effect was not statistically significant $(d=0.10 ; 95 \% \mathrm{CI}:-0.04,0.25$, $P=0.17)$. We observed no evidence of a significant moderation effect $(Q=6.16, \mathrm{df}=17, P=$ $0.17, I^{2}=0.00$ ) for any of the evaluated parameters, including study design, exercise intensity and delay period. For the study design, acute exercise was not associated with forgetting for between-subject designs $(d=0.02,95 \% \mathrm{CI}:-0.18-0.22, P=0.82)$ or within-subject designs $(d=0.19,95 \%$ CI: $-0.12-0.41, P=0.08)$. Results were also not statistically significant for moderate-intensity $(d=0.03,95 \% \mathrm{CI}:-0.28-0.34, P=0.84)$ or vigorous-intensity acute exercise $(d=0.13,95 \% \mathrm{CI}:-0.04-0.31, P=0.14)$. Similarly, results were not statistically significant for shorter delay periods $(d=0.06,95 \% \mathrm{CI}$ : $-0.16-0.28, P=0.58)$ or longer delay periods $(d=0.14,95 \% \mathrm{CI}:-0.06-0.33, P=0.18)$. 
a

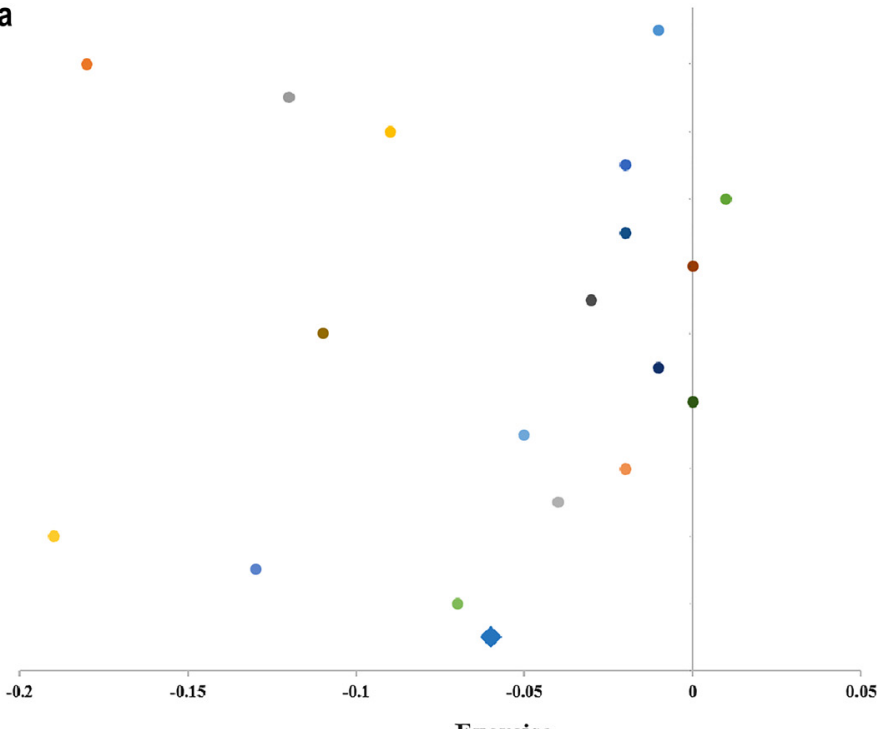

Allison Farris et al.

- James T. Haynes et al.

- Laruen Johnson et al._A

- Laruen Johnson et al._B

- Malina Austin et al.

- Lauren Ferguson et al._A

- Lauren Ferguson et al._B

- Breanna Wade et al.

- Danielle Yanes et al.

- Ali Siddiqui et al.

- Paul Loprinzi et al._A

- Paul Loprinzi et al._B

- Paul Loprinzi et al._C

- Paul Loprinzi et al._D

- Paul Loprinzi et al._E

- Jeremiah Blough et al.

- Emily Frith et al.

- Eveleen Sng et al.

-

Exercise

b

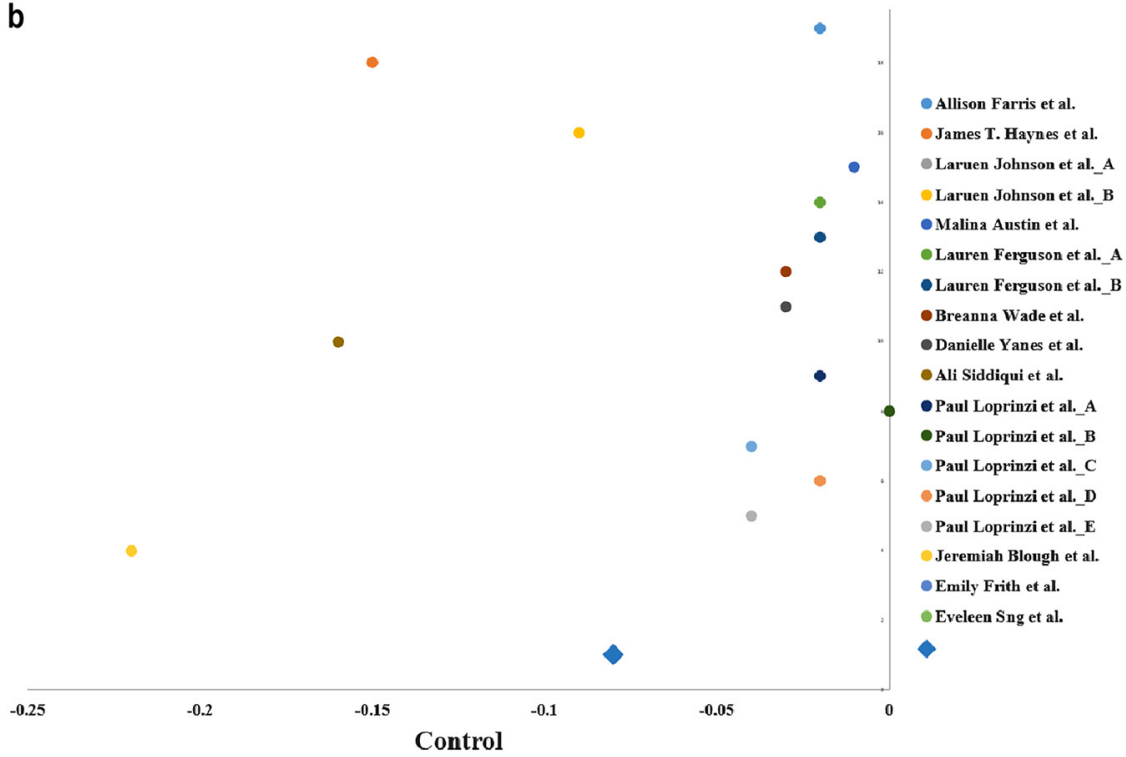

Fig. 2. (a) Change score (short-term memory minus long-term memory) in forgetting, expressed as a proportion, for the exercise condition. On average, acute exercise was associated with a $6 \%$ forgetting effect. (b) Change score (short-term memory minus long-term memory) in forgetting, expressed as a proportion, for the control condition. On average, the control condition was associated with a $7.5 \%$ forgetting effect. 

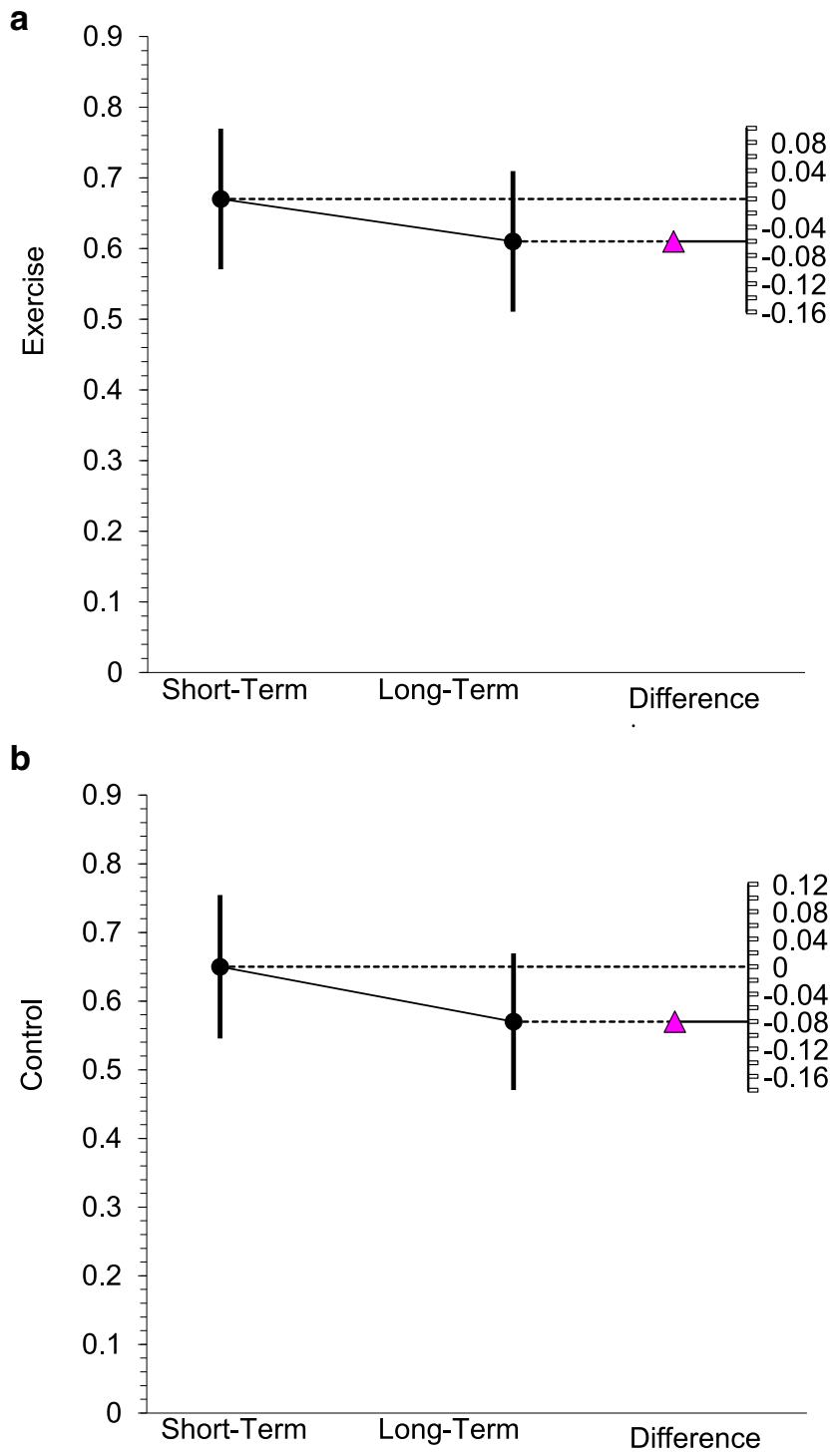

Fig. 3. (a). Short- and long-term memory outcomes (displayed as a proportion) for the exercise condition. Error bars represent 95\% CI. (b) Short- and long-term memory outcomes (displayed as a proportion) for the control condition. Error bars represent 95\% CI.

The regression intercept for the Egger's test (intercept $=-1.23, P=0.76$, two-tailed) was not statistically significant, indicating that there was no evidence of publication bias.

Fig. 2a and b display the short- and long-term memory outcomes (expressed as a proportion) for both the exercise (1a) and control conditions (1b). For the exercise condition, the proportion 
correct for the short- and long-term time periods, respectively, were $0.669(0.20)$ and 0.608 $(0.20)$. For the control condition, the proportion correct for the short- and long-term time periods, respectively, were $0.647(0.21)$ and $0.572(0.20)$. Lastly, the $\Delta$ score (forgetting) between short- and long-term memory was similar for exercise $(0.060)$ and control $(0.075)$ conditions. Fig. 3a (exercise) and $3 \mathrm{~b}$ (control) show the aggregate memory and forgetting results (averaged across the 12 experiments).

\section{DISCUSSION}

Previous experimental work demonstrates that acute exercise (i.e., walking/brisk walking on treadmill or jogging on treadmill) can enhance episodic memory function $[18,38]$; however, to date, few studies have examined the effects of acute exercise on forgetting. Our effect size (Fig. 1) results demonstrate that acute exercise occurring prior to memory encoding did not attenuate forgetting $(d=0.10,95 \% \mathrm{CI}:-0.04,0.25 ; P=0.17)$. Similarly, when evaluating the proportion of information that was correct for short-term and long-term memory, the acute exercise (6\%) and control scenarios $(7.5 \%)$ had a similar forgetting effect.

Of central interest of the present paper was whether acute exercise prior to memory encoding would attenuate forgetting. We observed no statistical evidence that acute exercise, prior to memory encoding, could attenuate forgetting. This finding aligns with the results from several of our other studies on this general topic. Pace and Loprinzi evaluated the effects of highintensity acute exercise on directed forgetting [39]. That is, after encoding List 1, and immediately before List 2, participants were instructed to either remember all of the words from List 1 or forget all of the words from List 1 . Those who were instructed to forget the List 1 words recalled more words from List 2. However, acute exercise did not have any effect on List 2 recall in the forgetting group. A study by Ferguson, Cantrelle and Loprinzi observed similar effects, but instead of evaluating this directed forgetting paradigm, the selective directed forgetting paradigm was used [40]. In support of these two studies, Cantrelle and Loprinzi also showed that acute exercise plays no effect in effectuating forgetting via the retrieval-induced forgetting paradigm [41]. Notably, these three studies were not included in the present analysis, as these three prior studies did not evaluate a short- and long-term memory assessment. Rather, they implemented existing intentional (directed forgetting and selective directed forgetting) and unintentional (retrieval-induced forgetting) forgetting paradigms.

The present findings of these 12 experiments, coupled with the findings from our 3 prior experiments on forgetting paradigms, suggests that acute exercise has a minimal, if any, effect on attenuating forgetting. However, prior work demonstrates that acute exercise can enhance both short- and long-term memory function [42]. Our present findings suggest that, for prior research on this topic, it is uncertain as to whether the exercise-induced effects on long-term memory are driven by exercise-induced improvements in short-term memory. That is, although acute exercise has been shown to enhance both short- and long-term memory, we did not observe any evidence that acute exercise affected the slope of decay between short- and longterm memory. As such, when evaluating the effects of acute exercise on long-term memory, future work on this topic should ensure that such effects are not an artifact of the effects of acute exercise on short-term memory. 
The present paper was not intended to be a meta-analysis of all existing studies on this specific topic. Rather, this paper reflects the work from our laboratory. As such, our findings will need to be interpreted in light of other work from other laboratories. Further, a limitation of this paper is the homogenous sample of young adults. Strengths of this paper, however, include the comprehensive assessment of acute exercise on forgetting (including 12 experiments from our lab) and evaluating multiple, potential moderators of this relationship.

In conclusion, our findings demonstrate that acute exercise is not [statistically] associated with forgetting. However, it would be worthwhile to investigate the effect of exercise on forgetting while incorporating a longer (e.g., 24-h recall) follow-up period to detect any potential changes in long-term memory. That is, it is possible that, in the evaluated studies, the relatively short delay period (e.g., 20-min) may not have been long enough to observe any exerciseinduced attenuation of forgetting. Additional work on this specific paradigm may also wish to consider incorporating the acute bout of exercise during the consolidation period. Emerging work demonstrates that acute exercise during the consolidation period is associated with longterm memory [42], and as such, this temporal positioning of exercise may attenuate forgetting. Further, future work on this emerging paradigm should be conducted among older adults and populations more susceptible to forgetting. Lastly, perhaps our null effects were a result of the exercise stimulus. As we discussed in the Introduction section, the prior research evaluating the effects of exercise on forgetting pathways (e.g., RAC1) employed chronic exercise paradigms. As such, chronic exercise (vs. acute exercise) may be required to attenuate forgetting. Future research should evaluate this possibility.

\section{ACKNOWLEDGMENTS}

We declare no conflicts of interest and no funding was used to prepare this manuscript.

\section{REFERENCES}

1. Loprinzi PD, Frith E, Edwards MK, Sng E, Ashpole N. The effects of exercise on memory function among young to middle-aged adults: systematic review and recommendations for future research. Am J Health Promot 2018; 32(3): 691-704.

2. Roediger HL, III, Weinstein Y, Agarwal PK. Forgetting: preliminary considerations. In: Forgetting. New York, NY, US: Psychology Press; 2010. p. 1-22.

3. Davis RL, Zhong Y. The biology of forgetting-a perspective. Neuron 2017; 95(3): 490-503.

4. Frankland PW, Josselyn SA, Kohler S. The neurobiological foundation of memory retrieval. Nat Neurosci 2019; 22(10): 1576-85.

5. Kandel ER, Dudai Y, Mayford MR. The molecular and systems biology of memory. Cell 2014; 157(1): 163-86.

6. Kitamura T, Ogawa SK, Roy DS, Okuyama T, Morrissey MD, Smith LM, et al. Engrams and circuits crucial for systems consolidation of a memory. Science 2017; 356(6333): 73-8.

7. Medina JH. Neural, cellular and molecular mechanisms of active forgetting. Front Syst Neurosci 2018; 12: 3-3.

8. Ferris LT, Williams JS, Shen CL. The effect of acute exercise on serum brain-derived neurotrophic factor levels and cognitive function. Med Sci Sports Exerc 2007; 39(4): 728-34. 
9. Park JK, Lee SJ, Kim TW. Treadmill exercise enhances NMDA receptor expression in schizophrenia mice. J Exerc Rehabil 2014; 10(1): 15-21.

10. Vaynman S, Ying Z, Gomez-Pinilla F. The select action of hippocampal calcium calmodulin protein kinase II in mediating exercise-enhanced cognitive function. Neuroscience 2007; 144(3): 825-33.

11. Heasman SJ Mammalian Rho GTPases: new insights into their functions from in vivo studies. Nat Rev Mol Cell Biol 2008; 9: 690-701.

12. Tejada-Simon MV. Modulation of actin dynamics by Rac1 to target cognitive function. J Neurochem 2015; 133(6): 767-79.

13. Dong T, He J, Wang S, Wang L, Cheng Y, Zhong Y. Inability to activate Rac1-dependent forgetting contributes to behavioral inflexibility in mutants of multiple autism-risk genes. Proc Natl Acad Sci U S A 2016; 113(27): 7644-9.

14. Shuai Y, Lu B, Hu Y, Wang L, Sun K, Zhong Y. Forgetting is regulated through rac activity in drosophila. Cell 2010; 140(4): 579-89.

15. Jiang L, Mao R, Zhou Q, Yang Y, Cao J, Ding Y, et al. Inhibition of Racl activity in the hippocampus impairs the forgetting of contextual fear memory. Mol Neurobiol 2016; 53(2): 1247-53.

16. Liu Y, Du S, Lv L, Lei B, Shi W, Tang Y, et al. Hippocampal activation of Rac1 regulates the forgetting of object recognition memory. Curr Biol 2016; 26(17): 2351-7.

17. Li Y, Zhao L, Gu B, Cai J, Lv Y, Yu L. Aerobic exercise regulates Rho/cofilin pathways to rescue synaptic loss in aged rats. PLoS One 2017; 12(2): e0171491.

18. Loprinzi PD, Frith E, Edwards MK, Sng E, Ashpole N. The effects of exercise on memory function among young to middle-aged adults: systematic review and recommendations for future research. American Journal of Health Promotion 2017; 32(3): 691-704.

19. Loprinzi P. The effects of exercise on long-term potentiation: a candidate mechanism of the exercise-memory relationship. OBM Neurobiol 2019; 3: 1-1.

20. Edwards MK, Blaha MJ, Loprinzi PD. Influence of sedentary behavior, physical activity, and cardiorespiratory fitness on the atherogenic index of plasma. J Clin Lipidol 2017; 11(1): 119-25.

21. Loprinzi PD, Frith E. A brief primer on the mediational role of BDNF in the exercise-memory link. Clin Physiol Funct Imaging 2019; 39(1): 9-14.

22. Loprinzi PD, Ponce P, Frith E. Hypothesized mechanisms through which acute exercise influences episodic memory. Physiol Int 2018; 105(4): 285-97.

23. Loprinzi PD. Forgetting may be useful, and exercise may help accentuate forgetting. J Cogn Behav Psychother Res 2019; 8(2): 128-30.

24. Cumming G. The new statistics: why and how. Psychol Sci 2013; 25(1): 7-29.

25. Garber CE. American College of Sports Medicine position stand. Quality and quantity of exercise for developing and maintaining cardiorespiratory, musculoskeletal and neuromuscular fitness in apparently healthy adults: Guidance for prescribing exercise. Med Sci Sports Exerc 2011; 43(7): 1334-59.

26. Farris A, Loprinzi PD. Experimental investigation of the effects of acute exercise on real-world ecological memory. J Sci Sport Exerc 2019; 1(1): 88-93.

27. Haynes JT, Frith E, Sng E, Loprinzi PD. Experimental effects of acute exercise on episodic memory function: considerations for the timing of exercise. Psychol Rep 2018; 122(5): 1744-54.

28. Johnson L, Loprinzi PD. The effects of acute exercise on episodic memory function among young university students: moderation considerations by biological sex. Health Promot Perspect 2019; 9(2): 99-104.

29. Austin M, Loprinzi PD. Acute exercise and mindfulness meditation on learning and memory: randomized controlled intervention. Health Promot Perspect 2019; 9(4): 314. 
30. Ferguson L, Loprinzi P. Experimental effects of acute moderate-intensity exercise on prioritized memory. J Neurobehav Sci 2019: 1.

31. Wade B, Loprinzi PD. The experimental effects of acute exercise on long-term emotional memory. J Clin Med 2018; 7(12).

32. Yanes D, Loprinzi PD. Experimental effects of acute exercise on iconic memory, short-term episodic, and long-term episodic memory. J Clin Med 2018; 7(6).

33. Siddiqui A, Loprinzi PD. Experimental investigation of the time course effects of acute exercise on false episodic memory. J Clin Med 2018; 7(7).

34. Loprinzi PD, Harris F, McRaney K, Chism M, Deming R, Jones T, et al. Effects of Acute exercise and learning strategy implementation on memory function. Medicina (Kaunas) 2019; 55(9).

35. Blough J, Loprinzi DP. Experimental manipulation of psychological control scenarios: implications for exercise and memory research. Psych 2019; 1(1).

36. Frith E, Sng E, Loprinzi PD. Randomized controlled trial evaluating the temporal effects of high-intensity exercise on learning, short-term and long-term memory, and prospective memory. Eur J Neurosci 2017; 46(10): 2557-64.

37. Sng E, Frith E, Loprinzi PD. Temporal effects of acute walking exercise on learning and memory function. Am J Health Promot 2018; 32(7): 1518-25.

38. Roig M, Nordbrandt S, Geertsen SS, Nielsen JB. The effects of cardiovascular exercise on human memory: a review with meta-analysis. Neurosci Biobehav Rev 2013; 37(8): 1645-66.

39. Pace ME, Loprinzi PD. High-intensity acute exercise and directed forgetting on memory function. Medicina (Kaunas) 2019; 55(8).

40. Ferguson L, Cantrelle J, Loprinzi P. Experimental effects of exercise on forgetting. OBM Integr Complement Med 2018; 3: 1-1.

41. Cantrelle J, Loprinzi P. Exercise and retrieval-induced forgetting. Psych 2019; 1(1).

42. Loprinzi PD, Blough J, Crawford L, Ryu S, Zou L, Li H. The temporal effects of acute exercise on episodic memory function: Systematic review with meta-analysis. Brain Sci 2019; 9(4): 87. 